\title{
Electron Kinetics in Radio-Frequency Atmospheric-Pressure Microplasmas
}

\author{
F. Iza* and J. K. Lee \\ Department of Electronic and Electrical Engineering, Pohang University of Science and Technology
}

M. G. Kong

Department of Electronic and Electrical Engineering, Loughborough University

(Received 28 February 2007; published 16 August 2007)

\begin{abstract}
The kinetic study of three radio-frequency atmospheric-pressure helium microdischarges indicates that the electron energy probability function is far from equilibrium, and three electron groups with three distinct temperatures are identified. The relative population of electrons in different energy regions is strongly time modulated and differs significantly from values recently reported from fluid analyses. It is also shown that a flux of energetic electrons $(\varepsilon>5 \mathrm{eV})$ that comprises up to $50 \%$ of the total electron flux can reach the electrodes. This energetic electron flux provides a new means of delivering energy to the electrodes and tuning the surface chemistry in atmospheric-pressure discharges. The three electron groups and the engineering of an energetic electron flux might open up a new paradigm in plasma-surface chemistry that has not been considered up until now.
\end{abstract}

PACS numbers: 52.80.Pi, 52.25.Jm, 52.65.Pp

Low-temperature atmospheric-pressure microplasmas constitute a new realm in plasma physics with potential economic and technological impact in many scientific disciplines. Their nonequilibrium character enables the use of plasmas in a broad range of applications without the need for vacuum systems. Recent research has focused on the use of microplasmas for material processing, displays, radiation sources, microsatellite propellers, biomedical applications, and environmental sensing [1-4]. Despite the rapid growth of the field, the physics of low-temperature high-pressure discharges remains not fully understood.

While traditional atmospheric-pressure plasmas operate close to thermal equilibrium (high gas temperature), it is now accepted that low-gas-temperature plasmas can also be realized. This is the case with microplasma sources. The reduced dimensions of a microdischarge provide a large surface-to-volume ratio, and favor large electric fields and steep space gradients. These characteristics enable a departure from thermodynamic equilibrium even at power levels of $\mathrm{MW} / \mathrm{cm}^{3}$. The reduced dimensions, however, make experimental characterization of the discharges very challenging. Probe diagnostics commonly used to characterize large-scale plasmas unavoidably perturb a microdischarge and optical diagnostics often cannot resolve the spatial structure of the discharge. Therefore, although diagnostics can provide estimates of some plasma parameters, precise time- and space-resolved kinetic information of charged particles has not been measured. However, since the energy probability function (EEPF) governs the reactivity and characteristics of a discharge, determining the electron kinetics in atmospheric-pressure microplasmas is fundamental in unraveling the underlying physics.

Given the experimental challenges, computer simulations provide a valuable alternative for studying microdischarges. Despite the nonequilibrium character of the low-temperature microplasmas, most studies found in the literature are based on fluid models. Kinetic effects not captured by hydrodynamic models, however, can be important in nonequilibrium discharges [5-11]. Thus, a kinetic analysis is required to validate existing fluid results $[2,12-15]$, determine the EEPF in microdischarges, and reveal the mechanisms leading to its formation. In this Letter, we address these issues by means of onedimensional $(1 \mathrm{~d} 3 \mathrm{v})$ particle-in-cell Monte Carlo collision (PIC-MCC) simulations (XPDP1 [16]).

Three rf atmospheric-pressure helium microdischarges are investigated. The discharges are sustained in between two parallel plate electrodes that are separated 200, 100, and $75 \mu \mathrm{m}$, respectively. A $13.56 \mathrm{MHz}-1 \mathrm{~A} / \mathrm{cm}^{2}$ current source is used to drive the discharges and the resulting peak voltage is $\sim 400 \mathrm{~V}$. The simulations track electrons and $\mathrm{He}^{+}$ions while the distribution of background neutrals is assumed to be time independent and uniform in space. Elastic, excitation, and ionization electron-neutral collisions are accounted for in the model. For ions, elastic scattering and charge exchange collisions are included. For simplicity and similar to what is normally done in fluid models, a constant ion-induced secondary electron emission coefficient of 0.1 is used in the study.

The time evolution of the electron density in the three microdischarges is shown in Fig. 1. The phase reference is taken at the time when the potential of the left-hand side electrode is at its minimum value. The peak densities are $17 \times, 2 \times$, and $0.6 \times 10^{19} \mathrm{~m}^{-3}$ for the 200, 100, and $75 \mu \mathrm{m}$ microdischarges, respectively. As the gap size shrinks, the sheaths occupy a larger portion of the discharge gap [Figs. 1(a)-1(c)]. When the sheaths are larger than half the discharge gap [Figs. 1(b) and 1(c)], the timeaveraged density profiles (not shown explicitly) do not form a quasineutral region. A similar observation was reported in [2]. It is noted, however, that instantaneously 


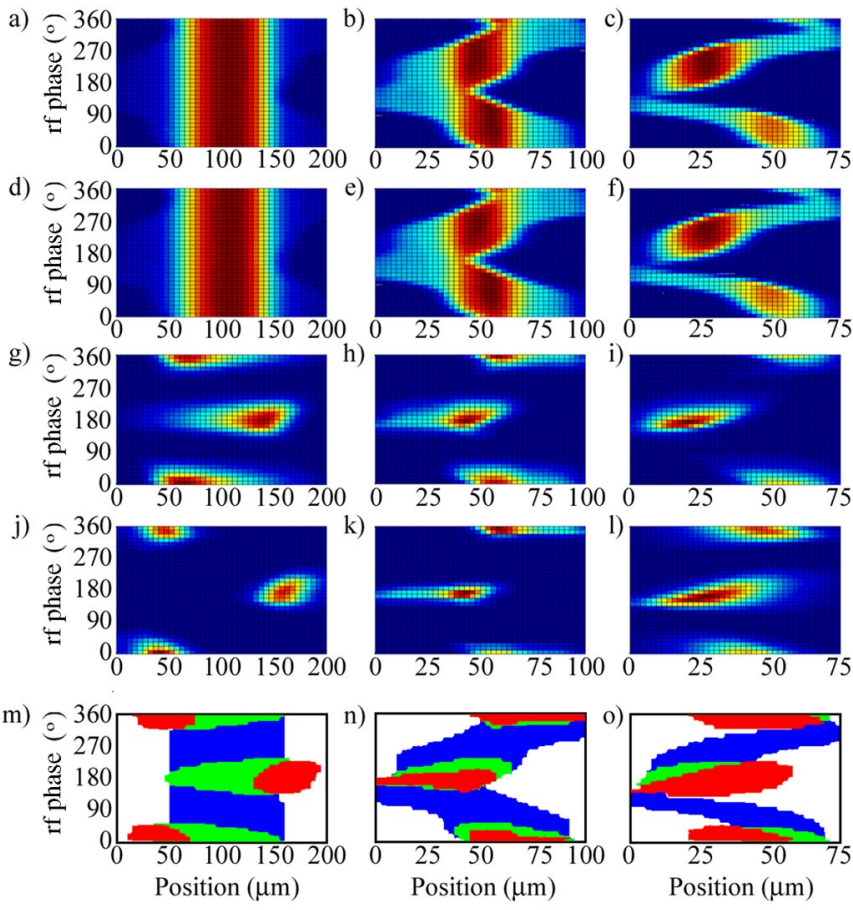

FIG. 1 (color online). Time evolution of the normalized electron density in $\mathrm{He}$ atmospheric microplasmas sustained in gaps of (a),(d),(g),(j),(m) $200 \mu \mathrm{m},(\mathrm{b}),(\mathrm{e}),(\mathrm{h}),(\mathrm{k}),(\mathrm{n}) 100 \mu \mathrm{m}$, and (c),(f),(i),(l),(o) $75 \mu \mathrm{m}$. (a)-(c) Total electron density. (d)(f) Normalized density of electrons with energy $\varepsilon<4 \mathrm{eV}$. (g) -(i) Normalized density of electrons with energy $4 \mathrm{eV}<\varepsilon<$ $20 \mathrm{eV}$. (j)-(1) Normalized density of electrons with energy $\varepsilon>$ $20 \mathrm{eV}$. (m)-(o) Superposition of the spatiotemporal density profiles of the three electron groups.

a region where quasineutrality still holds is observed in the simulations. This region moves back and forth across the gap at the driving $r f$ frequency and electrons bounce inside the instantaneous potential well at a higher frequency.

Since atmospheric-pressure discharges are highly collisional $(\lambda \sim 0.1 \mu \mathrm{m})$, heating of the discharges requires large electric fields, and collisionless and resonant heating often encountered in low-pressure rf discharges $[6,9,17]$ are typically negligible. Despite the high collisionality, electrons are not necessarily in local equilibrium with the electric field. The departure from local equilibrium has been recognized for secondary electrons accelerated in the sheaths and is typically accounted for in hybrid models $[2,12]$. In microdischarges, however, low-energy electrons may not be in local equilibrium either [18]. In fact, lowenergy electrons in the 100 and $75 \mu \mathrm{m}$ discharges can transit the confining ambipolar potential well without significant energy loss; i.e., they are in the nonlocal regime [5]. In other words, the energy relaxation length of lowenergy electrons is comparable to or larger than the width of the confining potential well $\left[\lambda_{\varepsilon}(\varepsilon)>L_{\text {well }}(\varepsilon)\right]$.

As a result of the differences in heating, cooling, generation, and loss of electrons of different energies, and the nonequilibrium character of the atmospheric-pressure microdischarges, the EEPF presents a three-temperature dis- tribution. This distribution contrasts with the Maxwellian, bi-Maxwellian, or Druyvesteyn EEPF $[7,8]$ typically observed in low-pressure discharges. The time evolution of the EEPFs for the three microdischarges of this study is shown in Fig. 2. It is noted that the relative population of electrons in different energy regions can differ by orders of magnitude from the values obtained with fluid models and that this difference could have a profound effect in the properties and reactivity predicted by those models. The effective electron temperature obtained in PIC-MCC simulations is $\sim 0.5 \mathrm{eV}$, whereas a hybrid model predicts temperatures of $\sim 4 \mathrm{eV}$ under similar conditions [2].

Three electron groups can be identified in the EEPFs (Fig. 2): low-energy $(\varepsilon<\sim 2 \mathrm{eV})$, midenergy ( $\sim 2 \mathrm{eV}<$ $\varepsilon<\sim 20 \mathrm{eV})$, and high-energy $(\varepsilon>\sim 20 \mathrm{eV})$. Their spatiotemporal evolution is shown in Figs. 1(d)-1(1). Lowenergy electrons are trapped by the ambipolar potential during the whole rf cycle and are inefficiently heated by the relatively weak electric field in the bulk. The $\sim 2 \mathrm{eV}$ threshold is set by the minimum of the confining potential during the collapse of the sheaths. On the other hand, the high-energy tail of the EEPF is populated by secondary electrons that have been accelerated in the sheaths. These are short-lived electrons that rapidly lose their energy once they reach the bulk plasma (in the absence of the sheath electric field, the electrons relax their energy in tens of picoseconds). Finally, midenergy electrons also result from the avalanches in the sheaths and do not last the whole rf cycle either. Besides relaxing their energy in a fraction of a rf cycle ( $\sim 1 \mathrm{~ns})$, midenergy electrons are also lost to the
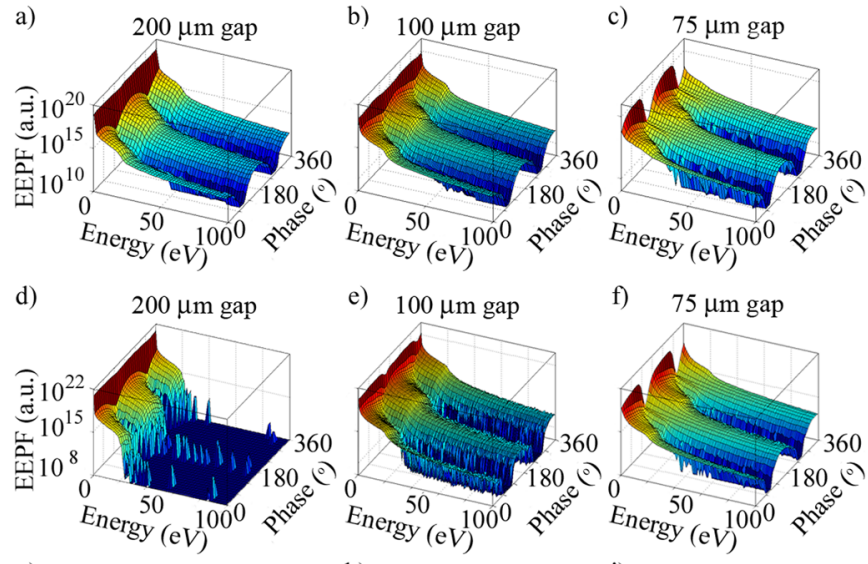

e) $100 \mu \mathrm{m}$ gap

f) $75 \mu \mathrm{m}$ gap
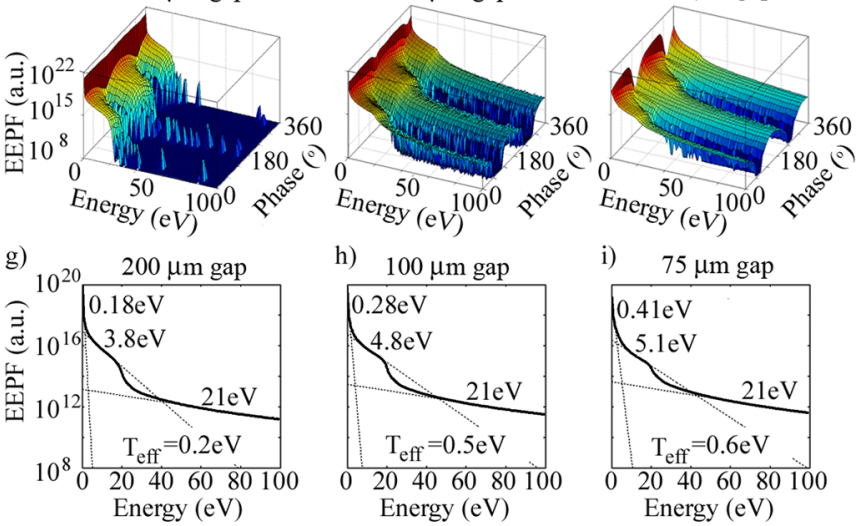

h) $100 \mu \mathrm{m}$ gap

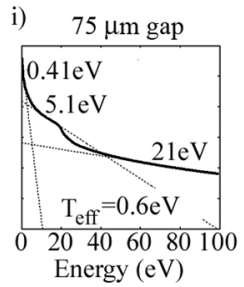

FIG. 2 (color online). Time evolution of the EEPF in three He microplasmas at atmospheric pressure driven at $1 \mathrm{~A} / \mathrm{cm}^{2}$. (a)(c) EEPF accounting for electrons in the whole gap. (d)(f) EEPF in the center of the discharge. (g)-(i) Space and time-averaged EEPF. 
electrodes when the sheaths collapse. The separation between the mid- and high-energy electrons at $\sim 20 \mathrm{eV}$ corresponds to the helium excitation threshold and as in lowpressure discharges, the "knee" in the EEPF is due to the faster energy relaxation of electrons in the inelastic energy range [5].

To elucidate the underlying physics leading to the formation of the EEPF, the evolution in time, space, and energy of a group of secondary electrons is presented in Fig. 3. Since the discharges are sustained in the $\gamma$ mode (justified later), the EEPFs shown in Fig. 2 can be interpreted as the superposition in time and space of partial EEPFs that result from the emission of secondary electrons at different times.

Let us consider a group of electrons emitted from the left-hand side electrode at an arbitrary phase [point 1 in Fig. 3(a)]. Initially, the secondary electrons are rapidly accelerated across the sheath and as a result their energy and temperature rapidly increase [see time evolution of the EEPF in Fig. 3(b)]. These electrons populate the highenergy tail of the EEPF shown in Fig. 2. As the electrons transit the sheath they initiate an ionization avalanche that lasts less than a nanosecond [Fig. 3(d)]. Depending on the phase at which the secondary electrons are emitted, the avalanche can generate up to 20 new electrons per secondary electron emitted; i.e., the electric field is large enough to cause the breakdown of the sheath. After the initial acceleration, some energetic electrons reach the opposite electrode and escape the discharge [point 2 in Figs. 3(a) and 3(c)], whereas others get trapped in the instantaneous ambipolar potential well. The high collisionality causes the high-energy tail (electrons with energy above the inelastic threshold) to relax in tens of picoseconds in the absence of a sufficiently large electric field. As a result, high-energy electrons rapidly disappear when they reach the bulk plasma [Fig. 3(b) -EEPF at 140-700 ps]. The remaining low- and midenergy electrons oscillate in the ambipolar potential losing their energy at a slower rate [Fig. 3(b)EEPF at $700 \mathrm{ps}-14 \mathrm{~ns}$ ]. When the sheaths collapse [point 3 in Figs. 3(a) and 3(c)], midenergy electrons either escape to the electrodes or lose their energy through elastic collisions with the background gas. It is noted that the short energy
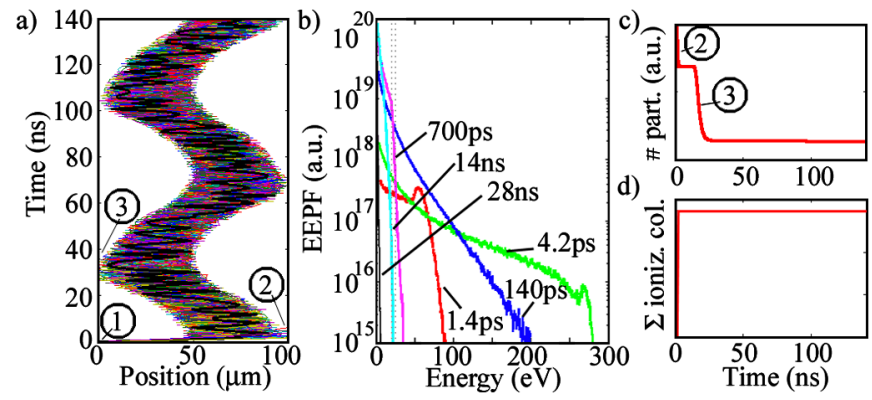

FIG. 3 (color online). Test electrons in a $100 \mu \mathrm{m}$ He microdischarge at atmospheric pressure. (a) Trajectories (subsampled).

(b) EEPF (c) Number of test electrons as a function of time.

(d) Number of ionizing collisions integrated over time. relaxation time of midenergy electrons ( $\sim 1 \mathrm{~ns})$ and the lack of significant heating at this time result in the disappearance of midenergy electrons during each collapse of the sheaths (they last $\sim 10 \mathrm{~ns}$ ). This is not the case in conventional low-temperature low-pressure rf discharges, where the energy relaxation time is orders of magnitude larger than the rf period. As seen in Fig. 3(b) at 28 ns, only low-energy electrons survive the collapse of the sheath and the high collisionality of the discharge prevents these lowenergy electrons from being subsequently heated up to the inelastic energy region (not shown explicitly).

Since the electron energy relaxation time $(<10 \mathrm{~ns})$ is significantly shorter than the rf period ( $\sim 80 \mathrm{~ns})$, the EEPF is strongly time modulated (Fig. 2). It is noted that the modulations of different energy groups are not in phase.

For discharges in large gaps ( $\geq 200 \mu \mathrm{m}$ ), the maximum sheath width is less than half the gap size and secondary electrons lose their energy before reaching the discharge center [Fig. 1(m)]. As a result, the EEPF in the center of the discharge recovers a conventional two-temperature distribution [Fig. 2(d)] typically observed in $\gamma$-mode discharges at low pressure [8].

The breakdown of the sheath, the inefficient heating of electrons towards the inelastic energy range, the loss of midenergy electrons every half rf cycle, and the spatiotemporal evolution of high-energy electrons suggest that the discharges are sustained in the $\gamma$ mode. In fact, it can be shown that for the three discharges $\int_{0}^{\tau_{\mathrm{rf}}} \Gamma_{i}(t) \gamma \zeta(t) d t=$ $\int_{0}^{\tau_{\mathrm{rf}}} \Gamma_{i}(t) d t$; i.e., ion losses are balanced by ionization events that originate from secondary electrons. Here, $\Gamma_{i}$ is the ion flux to one electrode, $\gamma$ the secondary electron emission coefficient $(0.1)$ and $\zeta$ the number of ionization events per secondary electron emitted. While $\alpha$ and $\gamma$ modes have been identified experimentally in atmospheric-pressure mm-size microdischarges [19,20], the authors are not aware of experimental data regarding discharges in smaller gaps. In our simulations, reducing the current density in the $75 \mu \mathrm{m}$ gap discharge results in the extinction of the discharge without a transition from the $\gamma$ to the $\alpha$ mode.

While particle kinetics in the discharge volume are important for determining reactions in the gas phase, the kinetics of particles arriving at the electrodes are vital in controlling the surface chemistry. At atmospheric pressure, collisions in the sheath limit the energy ions can acquire and ion-induced surface activation is normally not effective. Figure 4 shows the electron and ion fluxes to the lefthand side electrode as a function of time. As expected, the electron flux is strongly time-modulated with most electrons arriving at the electrode when the sheath around the electrode collapses. Although the maximum plasma density is obtained in the $200 \mu \mathrm{m}$ gap, the flux to the electrodes increases $\sim 5 \%$ and $\sim 10 \%$ as the gap size is reduced to $100 \mu \mathrm{m}$ and $75 \mu \mathrm{m}$, respectively. More significantly, the relative portion of energetic electrons $(\varepsilon>5 \mathrm{eV})$ increases by almost an order of magnitude (Fig. 4). Since 


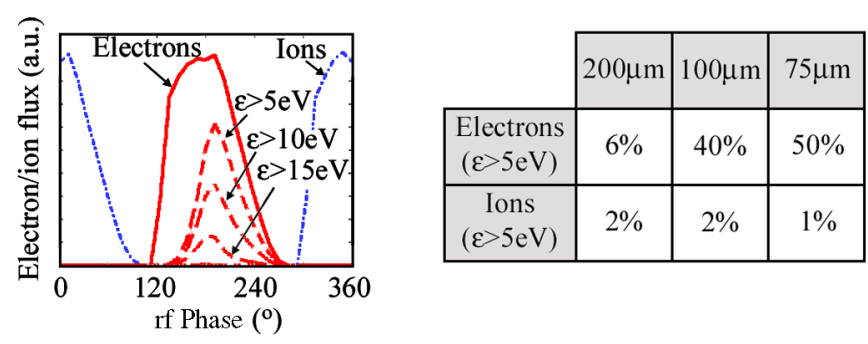

FIG. 4 (color online). Electron and ion fluxes to the left-hand side electrode as a function of time for a $100 \mu \mathrm{m}$ microdischarge. The table displays the time-averaged percentage of energetic particles striking the electrode for 200, 100, and $75 \mu \mathrm{m}$ discharges.

bond energies are typically of the order of a few $\mathrm{eV}$, one could engineer the gap size to optimize the arrival of energetic electrons and use this electron flux to control surface reactions.

Finally, it is noted that similarity rules can be used to compare the rf microdischarges with better-understood low-pressure plasmas. Scaling up the microdischarges while preserving $p d, \nu / \omega_{\mathrm{rf}}$ (number of collisions in one rf cycle), $E / p$ (energy gained between collisions), and $n_{e} / p^{2}$ (ratio of the electric field in the sheath to that in the bulk), however, results in unusually large plasmas with extremely low electron densities (e.g., 76 mTorr, $1 \mathrm{~m}$ gap, $1 \mathrm{kHz}, 10^{5} \mathrm{~cm}^{-3}$ ). As a result, differences arise between conventional low-pressure rf discharges operated in the $\gamma$-mode and the microdischarges described here. Most remarkably, the sheath width in the microplasmas is comparable to the gap size and energetic $\gamma$ electrons are a significant fraction of the total electron population. These characteristics contribute to the conspicuous threetemperature EEPF observed in the microplasmas and the energetic electron flux to the electrodes. In conventional low-pressure rf discharges, both the $\alpha$ and $\gamma$ modes are observed and an increase in the bulk plasma density accompanies the transition from the $\alpha$ to the $\gamma$ mode. This increase is due to the nonlocal spreading of the ionization caused by $\gamma$ electrons $[8,21]$. For the smallest microplasmas discussed in this Letter, however, the $\alpha$ mode could not be sustained and high-energy electrons can be considered to be in local equilibrium $\left(\lambda_{\varepsilon} \sim 7 \mu \mathrm{m} \ll L\right)$. It is also noted that contrary to what happens in low-pressure discharges [21,22], the ion flux to the electrodes (Fig. 4) and the ion density profile (not shown) are strongly time modulated both in the 100 and $75 \mu \mathrm{m}$ plasmas.

In conclusion, it has been shown that the EEPF in helium atmospheric-pressure rf microdischarges is far from equilibrium and thus the interpretation of simulation results obtained from fluid based models should be done cautiously. The EEPFs are strongly time modulated and three electron groups with three different temperatures have been identified. Because of the nonequilibrium character of the discharges, fluid simulations predict effective temperatures significantly larger than those predicted by PIC-
MCC models ( $\sim 4 \mathrm{eV}$ vs $\sim 0.5 \mathrm{eV}$ ). This difference could have a profound effect in the properties and reactivity predicted by fluid models. When the sheaths extend over half the discharge gap, energetic electrons generated in avalanches initiated by secondary electrons can reach the opposite electrode. Since ion-neutral collisions limit the energy ions acquire as they are accelerated across the sheaths, a flux of energetic electrons can be used to tailor surface chemistry in atmospheric-pressure discharges. Depending on the application, an electron flux could have the added benefit of transferring to the surface less momentum than an ion flux, thereby minimizing compressive stresses. The three distinct electron groups and the engineering of an energetic electron flux open up new paradigms in plasma-surface chemistry and in lowtemperature atmospheric-pressure rf discharges that have not been explored so far. The nonequilibrium character of the discharges shown in this Letter should motivate the kinetic study of microdischarges and more elaborated models shall be employed in the future.

The authors are grateful to Professor D. Graves (U.C. Berkeley), Professor H. C. Kim and Dr. S. J. You for their comments and fruitful discussions. This work was supported by the Korean Science \& Engineering Foundation.

*fiza@postech.ac.kr

[1] D. Janasek et al., Nature (London) 442, 374 (2006).

[2] J. J. Shi and M. G. Kong, Phys. Rev. Lett. 96, 105009 (2006).

[3] I.E. Kieft et al., Bioelectromagnetics (N.Y.) 25, 362 (2004).

[4] K. H. Becker, K. H. Schoenbach, and J. G. Eden, J. Phys. D: Appl. Phys. 39, R55 (2006).

[5] U. Kortshagen et al., Electron Kinetics and Applications of Glow Discharges (Plenum, New York, 1998).

[6] I. D. Kaganovich et al., Appl. Phys. Lett. 69, 3818 (1996).

[7] V. A. Godyak et al., Phys. Rev. Lett. 65, 996 (1990).

[8] V. A. Godyak et al., Phys. Rev. Lett. 68, 40 (1992).

[9] S. V. Berezhnoi et al., Plasma Phys. Rep. 24, 556 (1998).

[10] V. I. Kolobov, J. Phys. D: Appl. Phys. 39, R487 (2006).

[11] H. C. Kim et al., J. Phys. D: Appl. Phys. 38, R283 (2005).

[12] M. J. Kushner, J. Appl. Phys. 95, 846 (2004).

[13] Y. Sakiyama and D. B. Graves, J. Phys. D: Appl. Phys. 39, 3644 (2006).

[14] Q. Wang et al., J. Appl. Phys. 100, 023301 (2006).

[15] J. H. Seo et al., J. Appl. Phys. 100, 123302 (2006).

[16] J. P. Verboncoeur et al., J. Comput. Phys. 104, 321 (1993).

[17] G. Y. Park et al., Phys. Rev. Lett. 98, 085003 (2007).

[18] F. Iza et al., J. Appl. Phys. 98, 043302 (2005).

[19] J. J. Shi and M. G. Kong, J. Appl. Phys. 94, 6303 (2003).

[20] X. Yang et al., Plasma Sources Sci. Technol. 14, 314 (2005).

[21] S. V. Berezhnoi et al., IEEE Trans. Plasma Sci. 27, 1339 (1999).

[22] K.E. Orlov et al., IEEE Trans. Plasma Sci. 27, 1348 (1999). 